\title{
Overexpression of MATN3 predicts poor prognosis of gastric adenocarcinoma: based on TCGA database
}

\section{GuoLiang Zheng}

Liaoning Cancer Institute and Hospital

\section{Yan Zhao}

Liaoning Cancer Institute and Hospital

Zhichao Zheng ( $\sim$ drzhengzhichao1@163.com )

Cancer Hospital of China Medical University https://orcid.org/0000-0003-3064-976X

\section{Research article}

Keywords: MATN3, Gastric adenocarcinoma, The Cancer Genome Atlas, Prognosis, GSEA

Posted Date: February 13th, 2020

DOl: https://doi.org/10.21203/rs.2.23505/v1

License: (c) (i) This work is licensed under a Creative Commons Attribution 4.0 International License.

Read Full License 


\section{Abstract}

Background Matrilin-3 (MATN3) was previously reported to in the cartilage extracellular matrix and had a role in the development and homeostasis of cartilage and bone. We evaluated the role of MATN3 in gastric adenocarcinoma (GAC) using publicly available data from The Cancer Genome Atlas (TCGA). Methods The relationship between clinicapathologic features and MATN3 were analyzed with the Wilcoxon signed-rank test and logistic regression. Clinicopathologic variables associated with overall survival (OS) in TCGA patients using Cox regression and the Kaplan-Meier method. Gene Set Enrichment Analysis (GSEA) was performed using TCGA cohort. Results MATN3 overexpressed in GAC than that in

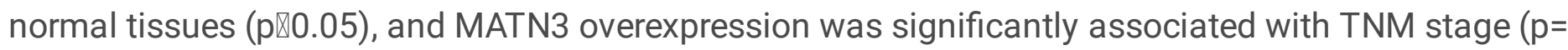
0.012), and T stage ( $\mathrm{p} \otimes 0.01$ ). Kaplan-Meier survival analysis showed that GAC with MATN3 - high had a worse prognosis than that with MATN3- low ( $\mathrm{p} \otimes 0.01)$. The univariate analysis revealed that MATN3- high correlated significantly with a poor OS (HR: 1.86; 95\% confidence interval [CI]: 0.82- 2.01; $p=0.014)$. The multivariate analysis revealed that MATN3 remained independently associated with OS, with a HR of 2.68

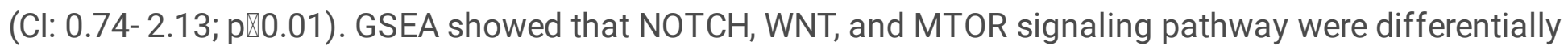
enriched in MANT3 high expression phenotype. Conclusion Overexpression of MATN3 may be a potential prognostic biomarker of poor survival in gastric cancer, Moreover, NOTCH, WNT, and MTOR signaling pathway may be the key pathway regulated by MATN3 in GAC.

\section{Introduction}

Gastric adenocarcinoma (GAC) remains one of the most widespread tumors in the world. It was the fifth most frequently diagnosed cancer and the third leading cause of cancer death according to the data of epidemiological survey in 2018 [1]. Recurrence and metastasis is the main cause of death in patients with GAC, which seriously hinders the success of treatment [2]. GAC is a complicated multi-step disease. The pathogenesis of GAC involves genomic changes, including gene amplification, deletion, insertion or mutation, which ultimately trigger a series of epigenetic and genetic changes. Therefore, the key to the prognosis and treatment of gastric cancer is to identify key genes and understand their molecular mechanisms. The matrilin (MATNs) are a family of oligomeric extracellular matrix (ECM) proteins, which consist at least four related proteins (matrilin-1 to 4). This family is thought to take part in the formation of filamentous networks in the extracellular matrices of various tissues. Matrilin-3 (MATN3), which is composed of one vWFA domain, four EGF-like domains, and a C-terminal coiled-coil domain, is the simplest member of the matrilin family $[3,4]$. Previous studies have found that MATN3 protein is present in the cartilage extracellular matrix and plays important roles in the development and homeostasis of cartilage and bone[5]. Mutations in the gene result in multiple epiphyseal dysplasia [6,7].Until now, the expression level of MATN3 in malignant tumors, especially in GAC, remains unclear.

Thus, this study aimed to investigate the prognostic value of MATN3 expression in GAC based on the TCGA datasets. GSEA was performed to gain further insight into the biological pathways involved in GAC pathogenesis related MATN3 regulatory network. 
Therefore, the purpose of this study was to evaluate the prognostic value of MATN3 expression in GAC based on TCGA, and further understand the GAC signaling pathway related to MATN3 regulation through GSEA.

\section{Materials And Methods}

\subsection{Downloading mRNA data and analysis}

The mRNA expression data and corresponding clinicopathologic data of the stomach projects were downloaded from TCGA website. We selected patients with pathological type of adenomas or adenocarcinomas. The expression differences for discrete variables were visualized using Boxplots[8]. Finally, The mRNA expression data of 406 samples with GAC and clinicopathologic data were analyzed (Table 1).

\subsection{GSEA}

In this study, the association between expression of MATN3 and biological pathway was performed using Gene Set Enrichment Analysis (GSEA v2.0)[9]. An ordered list of all genes according to their correlation with MATN3 expression was firstly generated, and samples from the TCGA datasets were divided into MATN3-high and -low groups. Default settings were used and permutation analysis (1000 times) was performed to determine thresholds for significance. False Discovery Rate (FDR) was calculated. When FDR is $₫ 0.25$, the gene set is considered significantly enriched.

\subsection{Statistical analysis}

All the statistical analyses were performed by $\mathrm{R}$ (v.3.6.0). The relationships between clinicopathologic characteristics and MATN3 were analyzed with logistic regression. Cox regression and Kaplan-Meier methods were used to analyze the relationship between clinicopathologic characteristics and OS. Multivariate Cox analysis was used to determine the impact of MATN3 expression level on OS. The samples were grouped to MATN3-high group and MATN3-low group by the median value of MATN3 expression.

\section{Results}

\subsection{Clinicopathologic features}

We downloaded 406 GAC samples with both clinical data and gene expression data based on TCGA in June 2019 with the median age at diagnosis 65.7 years old (Table 1). Male patients accounted for the majority (63.1\%).

The proportion of each pathological grade is $2.5 \%, 37.3 \%$ and $60.2 \%$ respectively for well, moderately and poorly differentiated. There were $56(14.7 \%), 118(31.1 \%), 167(43.9 \%)$ and $39(10.3 \%)$ patients with stage 
I, II, III and IV, respectively. 267 of 389 (68.6\%) cases had lymph node metastases. 27 of 388 (7\%) cases had distant metastases.

\subsection{Association of MATN3 expression level with clinicopathologic variables}

A total of 406 GAC samples from TCGA with MATN3 expression data were analyzed. METN3 in cancer tissues was significantly higher than that in normal tissues (Fig. 1A-B), and overexpression of MATN3 correlated significantly with the tumor clinical stage $(p=0.012)$ and T stage $(p \otimes 0.01)$ (Fig. 1C-D). Logistic regression analysis revealed that when the samples were grouped to MATN3-high group and MATN3-low group by the median value (2.34), overexpression of MATN3 was associated with poor clinicopathologic variables (Table 2). Overexpression of MATN3 in GAC was also significantly associated with pathologic TNM stage $(\mathrm{OR}=2.66(1.33-5.48)$ for II vs. I, OR = 1.97 (1.01-3.94) for III vs. I, OR = 2.45 (1.02-6.12) for IV vs. I), T stage (OR = $4.97(1.44-21.83)$ for T2 vs. T1, OR = 5.98 (1.9-26.45) for T3 vs. T1, OR = 6.26 (1.9228.49) for T4 vs. T1). These results indicated that GAC with MATN3 high expression was more likely to progress to more advanced stage than GAC with MATN3 low expression.

\subsection{Survival outcome}

Kaplan-Meier survival analysis showed that OS of GAC with MATN3-high was significantly lower than that with MATN3-low (pष0.01, Fig. 1E). Univariate analysis also showed that MATN3-high was significantly correlated with poor prognosis (hazard ratio [HR]: 1.06; 95\% confidence interval [CI]: 1.02 $1.11 ; p=0.0014)$. Other clinicopathologic variables, including age, Pathologic TNM Stage, lymph nodes and distant metastasis were also associated with poor prognosis (Table 3). Furthermore, multivariate analysis showed expression level of MATN3 was an independent prognostic factor, with a HR of 1.08 (Cl: 1.04-1.13, p囚0.01) (Table 4).

\subsection{Identification of MATN3-related signal pathways by GSEA}

We further used GSEA to confirm the correlation between MATN3 expression level and signal pathways. The Fig. 2 showed that P53, NOTCH, MAPK, and MTOR signaling pathways differentially enriched in MATN3 high expression phenotype.

\section{Discussion}

In this study, RNA-sequencing data based on TCGA were used for bioinformatics analysis, and it was found that the overexpression of MATN3 in GAC was associated with advanced clinical-pathological features (pathological TNM stage, T stage) and poor prognosis. We further used GSEA to study the 
function of MATN3 in GAC. GSEA showed that NOTCH, WNT, and mTOR signaling pathways differentially enriched in MATN3 high expression phenotype. It suggests that MATN3 may be a potential prognostic indicator for GAC.

MATN3, as a member of the matrilin protein family, is a noncollagenous extracellular matrix $[10,11]$. According to previous reports [5,11,12], it is mainly distributed in cartilage around the whole body, such as articular cartilage, costal cartilage, and tracheal cartilage. In primary human chondrocytes, MATN3 can induce the expression of MMP1, MMP3, MMP13, and COX2, indicating that MATN3 can regulate extracellular matrix degradation[12]. Existing data $[13,14]$ provide insights into the critical role of matrilin3 in inflammation, matrix degradation, and matrix formation in cartilage development and osteoarthritis. So far, although many studies about MATN3 have been limited to epiphyseal disease, few studies have been reported on other conditions such as malignant tumors. The potential role of MATN3 in GAC was the main research content of this study.

Zhou et al. [15] found that six gene signatures, including MATN3, could be used to predict recurrence in stage III and IV patients after surgery plus chemoradiotherapy. Wu et al. [16] also found MATN3 overexpression in GAC and its prognostic significance in OS of patients. In this study, MATN3 levels were significantly increased in stage IV patients and T4 patients, and multivariate analysis suggested that MATN3 was an independent prognostic factor $(p=0.000)$. Together, all these findings suggested that high expression of MATN3 could indicate a poor prognosis for GAC patients, and MATN3 might be a pivotal target gene involved in the process of GAC cell growth and metastasis. However, the molecular mechanisms underlying differentiation remained unclear.

In our study, we observed that MATN3 high expression phenotype was associated with the following signaling pathways: NOTCH, MAPK, and MTOR signaling pathways. MATN3 low expression phenotype was associated with P53 signaling which has been identified as one of the key signaling pathways that inhibit gastric cancer. Notch receptor plays different roles in the genesis and development of tumor[17]. The activation of Notch 1 and Notch 2 not only promotes the progression of gastric cancer[18,19], but also inhibits the invasion of gastric cancer[20,21]. NOTHC 3 contributes to glandular differentiation of gastric cancer[22], Notch4 promotes GC growth through the activation of Wnt1/ $\beta$-catenin signaling[23]. In addition, studies have confirmed that MTOR signaling pathway is activated in human gastric cancer and promotes cell proliferation. MTOR signaling pathway decreases after Notch inhibition, suggesting that MTOR is located downstream of Notch in gastric cancer cells[24]. In this paper, it is also proved that NOTCH, WNT and MTOR signaling pathways are active in gastric cancer cells with high expression of MATN3. However, the relationship between the expression of MATN3 and the above signaling pathways is reported for the first time, and its underlying regulatory mechanism needs to be further clarified.

However, using mRNA to predict protein expression was far from perfect [25]. This study did not evaluate the correlation between MATN3 mRNA expression and MATN3 protein expression, which was also the limitation. Further laboratory studies of gastric adenocarcinoma are needed in the future. 
In conclusions, MATN3 high expression may be a potential prognostic biomarker of poor survival in gastric adenocarcinoma. Moreover, NOTCH, WNT, and MTOR signaling pathways may be the key pathways regulated by MATN3 in GAC. Further experimental validation is needed to demonstrate the biological impact of MATN3.

\section{Abbreviations}

GAC = Gastric adenocarcinoma, GSEA = gene set enrichment analysis, MATN3 = Matrilin-3, NES = Normalized enrichment score, $\mathrm{NOM}-\mathrm{p}$ value $=$ Nominal $\mathrm{p}$ value, $\mathrm{OS}=$ overall survival, $\mathrm{TCG} A=$ The Cancer Genome Atlas.

\section{Declarations}

\section{Acknowledgments}

The results shown here are in whole or part based upon data generated by the TCGA Research Network: http://cancergenome.nih.gov/.

\section{Funding}

None.

\section{Author contributions}

Zheng GL collected the data, analyzed of data and wrote the manuscript; Zhao Y made critical revisions to the manuscript; Zheng ZC made substantial contributions to the conception, design, and coordination of the study and gave final approval of the version; all authors read and approved the final manuscript.

\section{Ethics approval and consent to participate}

Since this study only involves analysis of publically available database (TCGA) and does not contain any identifying patient information, the ethical approval of this study by Institutional review board (IRB) is not required. The TCGA data did not include the use of human subjects or personal identifying information. Thus, no informed consent was required for this part of the study.

\section{Competing interests}

The authors declare that they have no conflict of interest to this work. We declare that we do not have any commercial or associative interest that represents a conflict of interest in connection with the work submitted. 
No additional data are available.

\section{References}

[1] F. Bray, J. Ferlay, I. Soerjomataram, R.L. Siegel, L.A. Torre, A. Jemal, Global cancer statistics 2018: GLOBOCAN estimates of incidence and mortality worldwide for 36 cancers in 185 countries, CA Cancer J Clin 68 (2018) 394-424.

[2] G.P. Gupta, J. Massagué, Cancer metastasis: building a framework, Cell 127 (2006) 679-695.

[3] R. Wagener, B. Kobbe, M. Paulsson, Primary structure of matrilin-3, a new member of a family of extracellular matrix proteins related to cartilage matrix protein (matrilin-1) and von Willebrand factor, FEBS Lett. 413 (1997) 129-134.

[4] Y. Zhang, Z.K. Wang, J.M. Luo, K. Kanbe, Q. Chen, Multiple functions of the von Willebrand Factor A domain in matrilins: secretion, assembly, and proteolysis, J Orthop Surg Res 3 (2008) 21.

[5] A.R. Klatt, D.P. Nitsche, B. Kobbe, M. Mörgelin, M. Paulsson, R. Wagener, Molecular structure and tissue distribution of matrilin-3, a filament-forming extracellular matrix protein expressed during skeletal development, J. Biol. Chem. 275 (2000) 3999-4006.

[6] K.L. Chapman, G.R. Mortier, K. Chapman, J. Loughlin, M.E. Grant, M.D. Briggs, Mutations in the region encoding the von Willebrand factor A domain of matrilin-3 are associated with multiple epiphyseal dysplasia, Nat. Genet. 28 (2001) 393-396.

[7] A. Mabuchi, N. Haga, K. Maeda, E. Nakashima, N. Manabe, H. Hiraoka, et al., Novel and recurrent mutations clustered in the von Willebrand factor A domain of MATN3 in multiple epiphyseal dysplasia, Hum. Mutat. 24 (2004) 439-440.

[8] J. Kruppa, K. Jung, Automated multigroup outlier identification in molecular high-throughput data using bagplots and gemplots, BMC Bioinformatics 18 (2017) 232.

[9] A. Subramanian, P. Tamayo, V.K. Mootha, S. Mukherjee, B.L. Ebert, M.A. Gillette, et al., Gene set enrichment analysis: a knowledge-based approach for interpreting genome-wide expression profiles, Proc. Natl. Acad. Sci. U.S.A. 102 (2005) 15545-15550.

[10] A.R. Klatt, A.K. Becker, C.D. Neacsu, M. Paulsson, R. Wagener, The matrilins: modulators of extracellular matrix assembly, Int. J. Biochem. Cell Biol. 43 (2011) 320-330.

[11] R. Wagener, H.W. Ehlen, Y.P. Ko, B. Kobbe, H.H. Mann, G. Sengle, et al., The matrilins--adaptor proteins in the extracellular matrix, FEBS Lett. 579 (2005) 3323-3329. 
[12] A.R. Klatt, G. Klinger, B. Paul-Klausch, G. Kühn, J.H. Renno, R. Wagener, et al., Matrilin-3 activates the expression of osteoarthritis-associated genes in primary human chondrocytes, FEBS Lett. 583 (2009) 3611-3617.

[13] C.T. Jayasuriya, F.H. Zhou, M. Pei, Z. Wang, N.J. Lemme, P. Haines, et al., Matrilin-3 chondrodysplasia mutations cause attenuated chondrogenesis, premature hypertrophy and aberrant response to TGF- $\beta$ in chondroprogenitor cells, Int J Mol Sci 15 (2014) 14555-14573.

[14] M.S. Muttigi, I. Han, H.K. Park, H. Park, S.H. Lee, Matrilin-3 Role in Cartilage Development and Osteoarthritis, Int J Mol Sci 17 (2016)

[15] Y.Y. Zhou, Y.T. Kang, C. Chen, F.F. Xu, H.N. Wang, R. Jin, Combination of TNM staging and pathway based risk score models in patients with gastric cancer, J. Cell. Biochem. 119 (2018) 3608-3617.

[16] P.L. Wu, Y.F. He, H.H. Yao, B. Hu, Martrilin-3 (MATN3) Overexpression in Gastric Adenocarcinoma and its Prognostic Significance, Med. Sci. Monit. 24 (2018) 348-355.

[17] Z. Aburjania, S. Jang, J. Whitt, R. Jaskula-Stzul, H. Chen, J.B. Rose, The Role of Notch3 in Cancer, Oncologist 23 (2018) 900-911.

[18] T.S. Yeh, C.W. Wu, K.W. Hsu, W.J. Liao, M.C. Yang, A.F. Li, et al., The activated Notch1 signal pathway is associated with gastric cancer progression through cyclooxygenase-2, Cancer Res. 69 (2009) 50395048 .

[19] Y.C. Tseng, Y.H. Tsai, M.J. Tseng, K.W. Hsu, M.C. Yang, K.H. Huang, et al., Notch2-induced COX-2 expression enhancing gastric cancer progression, Mol. Carcinog. 51 (2012) 939-951.

[20] W. Zhou, X.Q. Fu, L.L. Zhang, J. Zhang, X. Huang, X.H. Lu, et al., The AKT1/NF-kappaB/Notch1/PTEN axis has an important role in chemoresistance of gastric cancer cells, Cell Death Dis 4 (2013) e847.

[21] L.Y. Guo, Y.M. Li, L. Qiao, T. Liu, Du YY, J.Q. Zhang, et al., Notch2 regulates matrix metallopeptidase 9 via PI3K/AKT signaling in human gastric carcinoma cell MKN-45, World J. Gastroenterol. 18 (2012) 72627270 .

[22] H. Kang, H.J. An, J.Y. Song, T.H. Kim, J.H. Heo, D.H. Ahn, et al., Notch3 and Jagged2 contribute to gastric cancer development and to glandular differentiation associated with MUC2 and MUC5AC expression, Histopathology 61 (2012) 576-586.

[23] C. Qian, F. Liu, B. Ye, X. Zhang, Y. Liang, J. Yao, Notch4 promotes gastric cancer growth through activation of Wnt1/ $\beta$-catenin signaling, Mol. Cell. Biochem. 401 (2015) 165-174.

[24] E.S. Hibdon, N. Razumilava, T.M. Keeley, G. Wong, S. Solanki, Y.M. Shah, et al., Notch and mTOR Signaling Pathways Promote Human Gastric Cancer Cell Proliferation, Neoplasia 21 (2019) 702-712. 
[25] Y. Guo, P. Xiao, S. Lei, F. Deng, G.G. Xiao, Y. Liu, et al., How is mRNA expression predictive for protein expression? A correlation study on human circulating monocytes, Acta Biochim. Biophys. Sin. (Shanghai) 40 (2008) 426-436.

\section{Tables}

\begin{tabular}{|c|c|c|}
\hline Clinical characteristics & Total $(\mathrm{N}=406)$ & $\%$ \\
\hline Age at diagnosis & $65.7 \llbracket 30-90 \square$ & \\
\hline \multicolumn{3}{|l|}{ Gender } \\
\hline male & 256 & 63.1 \\
\hline female & 150 & 36.9 \\
\hline \multicolumn{3}{|l|}{ Histologic Grade } \\
\hline well & 10 & 2.5 \\
\hline moderate & 149 & 37.3 \\
\hline poor & 240 & 60.2 \\
\hline \multicolumn{3}{|l|}{ Pathologic TNM Stage } \\
\hline I & 56 & 14.7 \\
\hline II & 118 & 31.1 \\
\hline III & 167 & 43.9 \\
\hline IV & 39 & 10.3 \\
\hline \multicolumn{3}{|l|}{$\mathrm{T}$} \\
\hline $\mathrm{T} 1$ & 23 & 5.8 \\
\hline T2 & 85 & 21.5 \\
\hline T3 & 185 & 46.7 \\
\hline $\mathrm{T} 4$ & 103 & 26 \\
\hline \multicolumn{3}{|l|}{$\mathrm{N}$} \\
\hline N0 & 122 & 31.4 \\
\hline N1 & 109 & 28 \\
\hline N2 & 80 & 20.6 \\
\hline N3 & 78 & 20 \\
\hline \multicolumn{3}{|l|}{ Distant metastasis } \\
\hline positive & 361 & 93 \\
\hline negative & 27 & 7 \\
\hline
\end{tabular}

Table 1. The characteristics gastric adenocarcinoma patient based on TCGA.

\begin{tabular}{|c|c|c|c|}
\hline Age at diagnosis (continuous) & 342 & $0.89(0.59-1.38)$ & 0.626 \\
\hline Gender $\square$ male vs. female $\square$ & 344 & $1.02(0.66-1.58)$ & 0.944 \\
\hline Histologic grade (well/moderate vs. poor) & 337 & $1.67(0.39-8.4)$ & 0.497 \\
\hline \multicolumn{4}{|l|}{ Pathologic TNM stage } \\
\hline II vs. I & 152 & $2.66(1.33-5.48)$ & 0.006 \\
\hline III vs.I & 185 & $1.97(1.01-3.94)$ & 0.049 \\
\hline IV vs. I & 85 & $2.45(1.02-6.12)$ & 0.047 \\
\hline \multicolumn{4}{|l|}{$\mathrm{T}$} \\
\hline T2 vs. T1 & 94 & $4.79(1.44-21.83)$ & 0.02 \\
\hline T3 vs. T1 & 176 & $5.98(1.9-26.45)$ & 0.0058 \\
\hline T4 vs. T1 & 104 & $6.29(1.92-28.49)$ & 0.0055 \\
\hline \multicolumn{4}{|l|}{$\mathrm{N}$} \\
\hline N1 vs. N0 & 197 & $0.97(0.55-1.71)$ & 0.907 \\
\hline N2 vs. N0 & 173 & $1.75(0.68-2.29)$ & 0.478 \\
\hline N3 vs. N0 & 167 & $1.19(0.64-2.22)$ & 0.591 \\
\hline Distant metastasis (positive vs. negative) & 322 & $0.88(0.43-2.20)$ & 0.431 \\
\hline
\end{tabular}

Table 2. MANT3 expressiona associated with clinical pathological characteristics (logistic regression). 


\begin{tabular}{lcccc}
\hline Clinicopathologic variables & HR & HR.95L & HR.95H & p-value \\
\hline Age at diagnosis & 1.93 & 1.01 & 1.99 & 0.004 \\
\hline Gender & 1.49 & 0.98 & 2.29 & 0.062 \\
\hline Histologic grade & 1.25 & 0.86 & 1.84 & 0.252 \\
\hline Pathologic TNM Stage & 1.91 & 1.19 & 1.91 & 0.0005 \\
\hline T & 1.58 & 0.99 & 1.63 & 0.0503 \\
\hline $\mathrm{N}$ & 1.85 & 1.05 & 1.49 & 0.0133 \\
\hline M & 2.07 & 1.07 & 3.98 & 0.0302 \\
\hline MATN3 & 1.86 & 0.82 & 2.01 & 0.0014 \\
\hline
\end{tabular}

Table 3. Univariate analysis of the relationship between OS and clinicopathologic variables in TCGA patients using Cox regression

\begin{tabular}{lcccc}
\hline Clinicopathologic variables & HR & HR.95L & HR.95H & p-value \\
\hline Age at diagnosis & 2.05 & 0.82 & 2.07 & $3.11 \mathrm{E}-05$ \\
\hline Gender & 1.52 & 0.97 & 2.36 & 0.0639 \\
\hline Histologic grade & 1.36 & 0.89 & 2.05 & 0.1474 \\
\hline Pathologic TNM Stage & 1.42 & 0.89 & 2.25 & 0.13387 \\
\hline T & 1.14 & 0.81 & 1.61 & 0.45651 \\
\hline $\mathrm{N}$ & 1.04 & 0.79 & 1.36 & 0.7846 \\
\hline M & 2.19 & 0.92 & 5.17 & 0.0735 \\
\hline MATN3 & 2.68 & 0.74 & 2.13 & $8.77 \mathrm{E}-05$ \\
\hline
\end{tabular}

Table 4. Multivariate analysis of the relationship between OS and clinicopathologic variables in TCGA patients

\section{Figures}
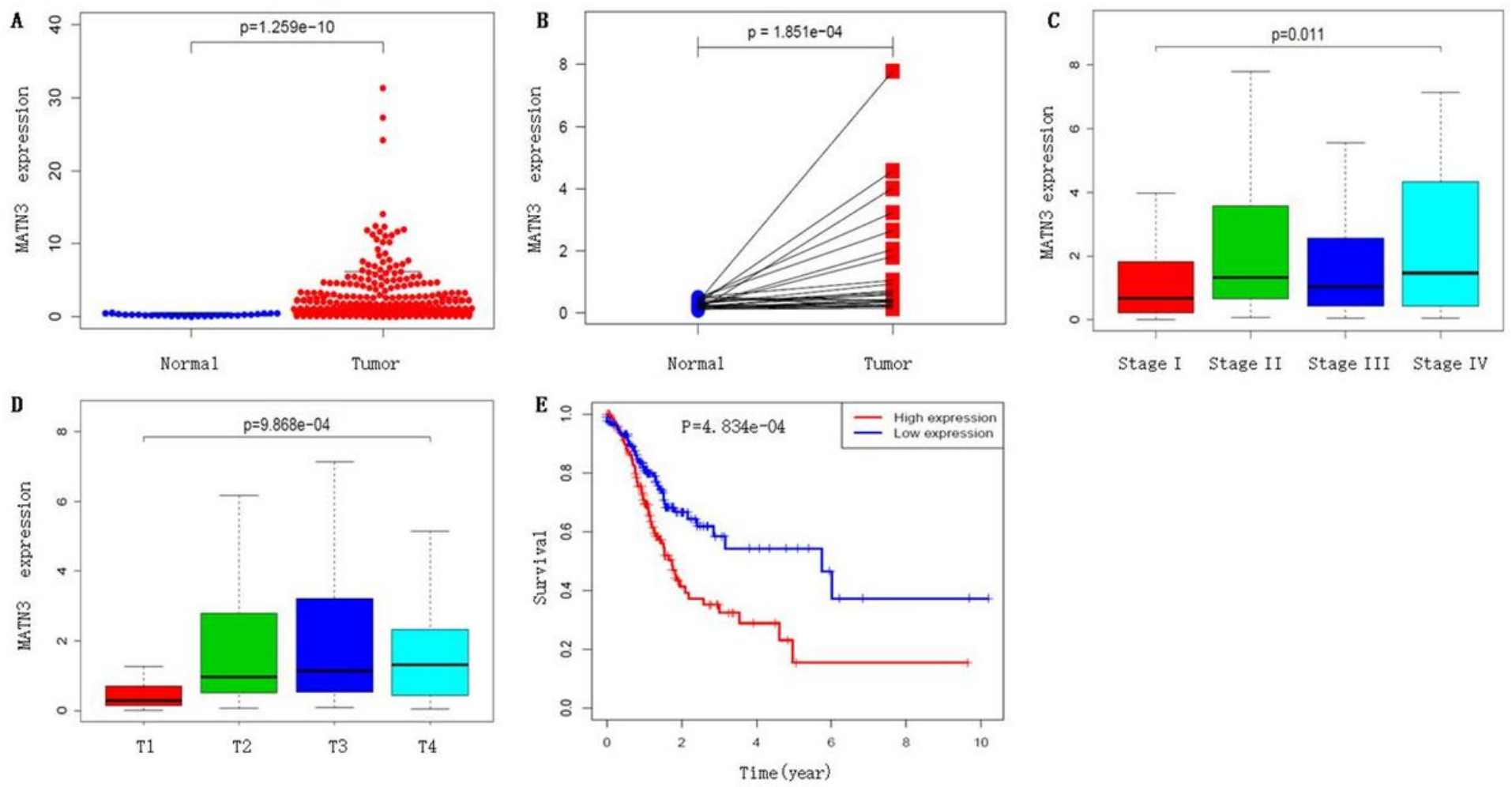
Figure 1

Association of MATN3 expression level with clinicopathologic variables

A Enrichment plot: KEGG_P53_SIGNALING_PATHWAY

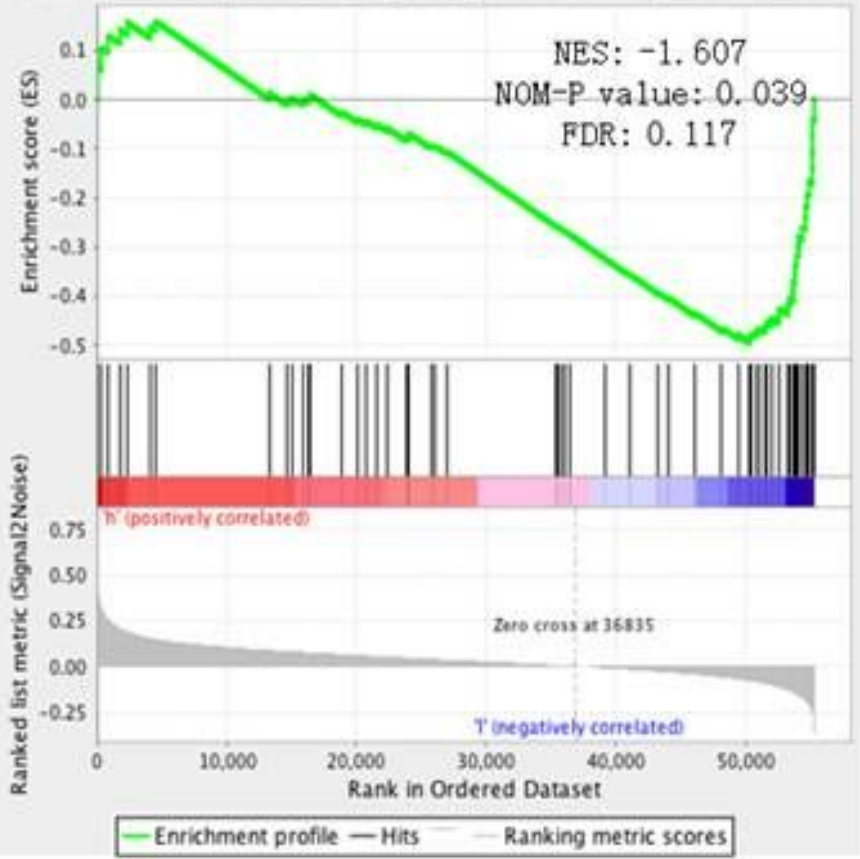

C Enrichment plot: KEGG_WNT_SIGNALING_PATHWAY

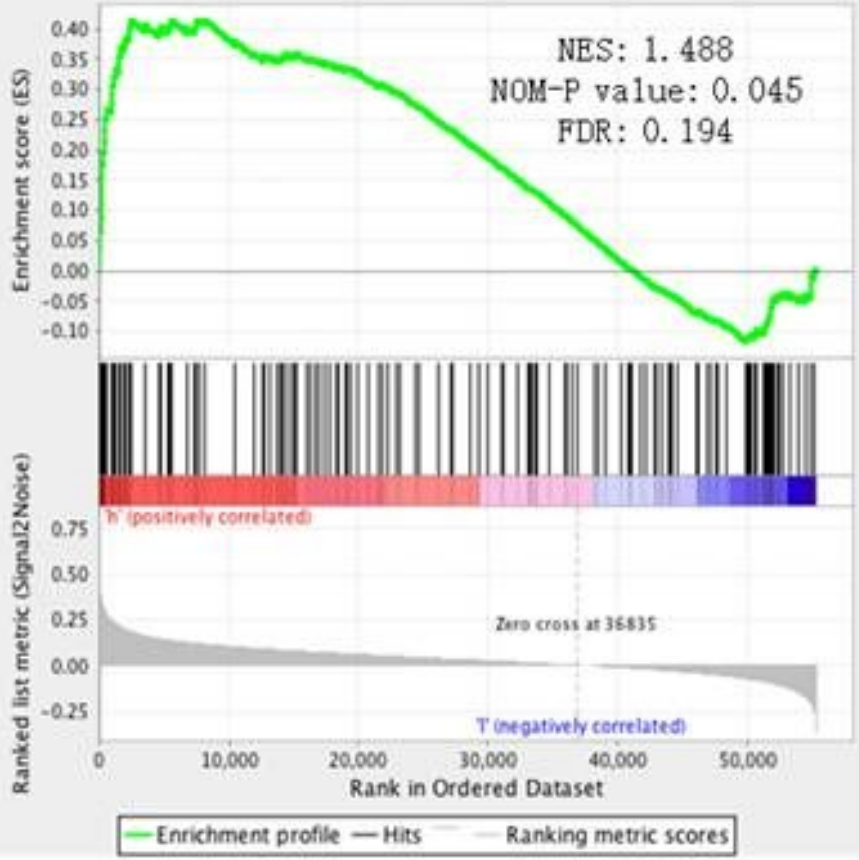

B Enrichment plot: KEGG_NOTCH_SIGNALING_PATHWAY

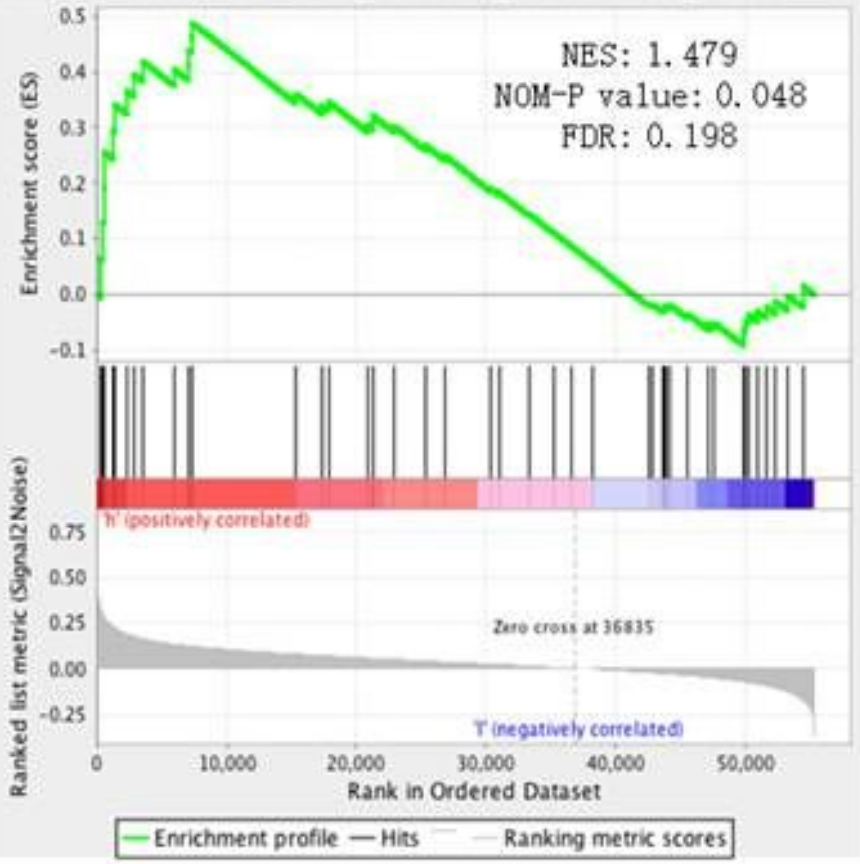

D Enrichment plot: KEGG_MTOR_SIGNALING_PATHWAY

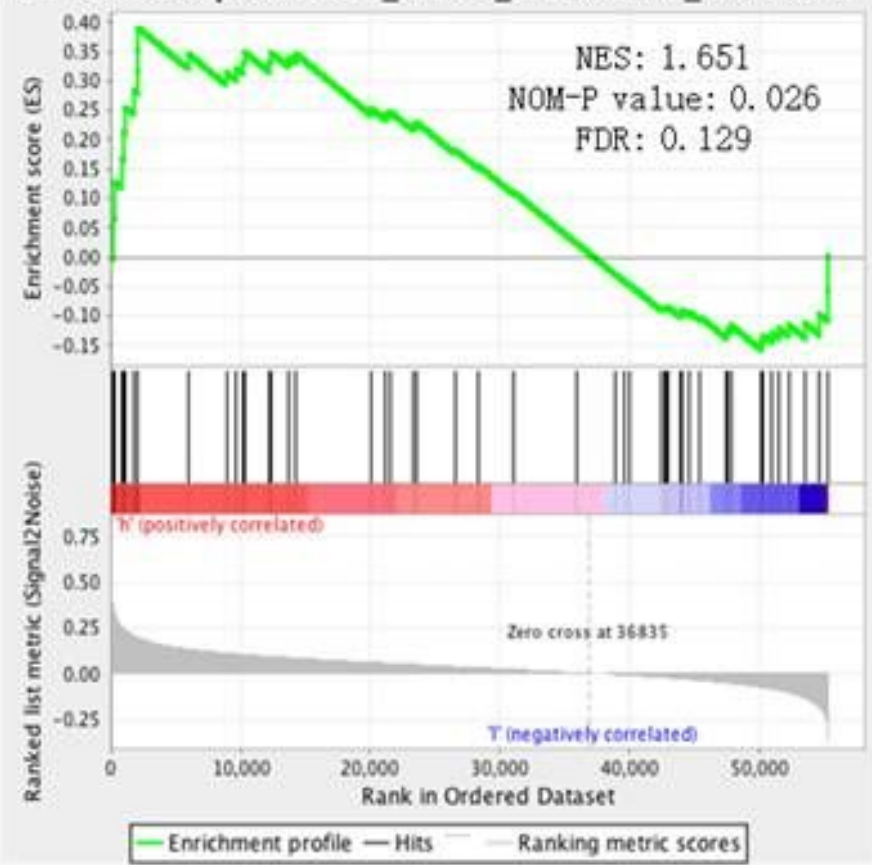

Figure 2

Identification of MATN3-related signal pathways by GSEA 